\title{
A Visual Interface for a Collection of Computer-Generated Narratives
}

\author{
Taisuke Akimoto *, Takashi Ogata ${ }^{\dagger}$
}

\begin{abstract}
Lately, automatic content generation has been receiving increasing attention, and studies pertaining to narrative generation systems have great scope of application in this field. A fundamental issue in designing applications of narrative generation technology is developing a connection between a narrative generation program and people. In this paper, we propose a visual interface for providing various computer-generated narratives to the user. The narrative generation program was also developed by us previously. The interface is used to arrange and visualize narratives into a two-dimensional space on the basis of their structural features. Users will be able to explore and browse narratives by using the visualized objects based on their own values or objectives. We have shown several visualization examples to help in discussing the availability of the interface. In addition, we have presented a new line of research, brought to light by this proposal - the vision of a co-creative connection between narrative generation systems and people, in particular.
\end{abstract}

Keywords: computational creativity, interface, narrative generation system, visualization

\section{Introduction}

Narrative generation is a complex but essential part of artificial intelligence and computational creativity; it has been a subject of interest since the '70s [1]. Although interactive narrative systems are currently a major genre of narrative content using computer technologies [2], there are very few applied studies on fully automatic narrative generation technologies. However, automatic content generation has been receiving increasing attention lately, and narrative generation systems have great potential in this field.

A drawback of computational narrative generation is the diversity and uncertainty of the value standards of a narrative. The value of a narrative varies with various factors, such as individual value, purpose or situation of the reception or use, and historical or cultural background. Hence, it is difficult to computationally define and control the value of narratives. However, it is necessary to computationally define and control the value of narratives for designing applicative narrative generation systems.

${ }^{*}$ The University of Electro-Communications, Tokyo, Japan

$\dagger$ Iwate Prefectural University, Iwate, Japan 
In most of the previous narrative generation systems, the value standards or the domain of generating narratives was strongly narrowed in the design phase. For instance, although BRUTUS [3] by Bringsjord and Ferrucci was a relatively large-scale narrative generation system, it was limited to generating stories on the theme of betrayal. Several studies have stated various criteria to establish a "good" narrative for evaluating a system's outputs or controlling the narrative generation process. For instance, Pérez y Pérez proposed an automatic evaluation program for computer-generated stories [4]. This program evaluates a story in terms of its novelty, coherence, and interestingness, by analyzing the story's characteristics, mainly dramatic tension, the precondition of each event, and its uniqueness from existing stories in the system's database. McIntyre and Lapata proposed a method for plot generation by applying a genetic algorithm [5]. A fitness function for coherence is used for selecting the most adequate story from a variety of candidate stories. Kartal et al. proposed a method for searching an optimal story from a large number of possible stories in a planning-based story generation technique [6]. Two evaluation criteria for stories - user-defined goals and believability — are applied for narrowing the solution space. A common trait of the above studies is that they formalize the problem of narrative generation as a type of optimization based on some criteria.

However, we take a different approach toward the aforementioned problem. In our design concept, the main role of the narrative generation system is to produce diverse narratives. The user searches for valuable narratives from the products based on one's own values. For this, an interface that allows the user to find valuable or required narratives from all generated narratives is required in addition to a mechanism that automatically produces various narratives. From this perspective, we propose a visual interface for forming a connection between many computer-generated narratives and the user. The key idea of the design is to use a narrative generation system as an exploration of valuable narratives. We employ visualization as the main method. In particular, the interface arranges the visualized objects of narratives into a two-dimensional space. In the narrative generation program, we employ the narrative discourse generation system proposed in 2012 [7]. The generation and interface parts are implemented using Common Lisp and Processing programming languages, respectively. The purpose of this paper is to discuss the above concept by presenting its first implementation. On the other hand, [8] has provided, using the implemented system presented in this paper, an experimentation-based discussion on the availability and future research directions of that concept.

An advantage of this approach compared with previous optimization-oriented studies is that the system allows multiple uses by various types of users. In general, possible uses of narrative generation systems or their output narratives include entertaining humans and providing ideas or resources for narrative creation. For entertaining humans, the system needs to provide narratives that match individual preferences or requirements. Although it is better for the system to promptly generate a suitable narrative for an individual requirement, the user probably has no clear needs on the narratives, or hopes to find unexpectedly good narratives. Likewise, when the user explores ideas for creating an original narrative, the requirements might be unclear and depend on the creation purpose, e.g., whether it is a story for a manga or a scenario for an experimental film. In the above types of situations, the interactive exploration of many and various computer-generated narratives is a valid approach, and this provides a new perspective on the problem of computational narrative generation. However, this approach is insufficient for cases that require outputting one narrative with some basis. For example, when a humanoid robot tells a narrative in the interaction with a human, some sort of optimization method is required, e.g., a mechanism for generating an adequate narrative or a basis for selecting an optimal narrative from many, depending on the context or situation of the interaction.

The discourse generation system is developed for a component of our integrated narrative 
generation system (INGS) $[9,10]$, which consists of three generative modules for "story" (the content information of a narrative), "discourse" (the structural aspect of how a story is expressed), and "expression" by using language, picture, and music. INGS was developed based on the concept of "expanded literary theory" [11] that tackles the design and development of narrative generation systems and mechanisms by using information technology, depending on social narrative generation models and modern literary theories, such as narratology. As INGS aims at the methodological diversity of narrative generation, we have also been attempting a different approach from the one described in this paper, i.e., the development of a technique to precisely create a single narrative through an incremental generation process ${ }^{*}$. Thus, the use of a visual interface for diverse narrative production by the system and narrative-selection by users is one of the potential uses of INGS. In this sense, although the implemented interface is specialized to suit the discourse module, the basic method is applicable to an interface for INGS itself. However, from a broader perspective, this paper explores a new form of connection between a narrative generation system and its users. Although the proposed system runs on a personal computer, it will be applied to new web-based services driven by narrative generation functions, such as distribution of computer-generated narratives.

\section{Outline of the Narrative Discourse Generation System}

Although this paper does not explain the details of the discourse generation system, it outlines its functions (for details on the system, refer to [7, 10]). The system generates a series of different narrative discourse structures from an input story structure that represents a temporal sequence of events. Both story and discourse are described as conceptual representations in the form of a tree consisting of "events" and "relations." An event is the basic unit of a story or discourse, whereas a relation semantically combines the child nodes, i.e., the events or sub-structures. The system includes a simple natural language generator, which transforms a conceptual representation into Japanese text. For instance, an event described as “(event 誘拐する< abduct $>$ (agent 蛇< snake>) (counter-agent 皇女 $<$ princess $>$ ) (location 庭 $<$ garden $>$ ))” is transformed to “蛇が皇女を誘拐 した $<\mathrm{A}$ snake abducted a princess $>$ " (we have annotated English translations in the angle brackets).

Discourse generation refers to structural transformations from a story structure to a discourse structure. The system equips 13 transformation techniques implemented based on a computational refinement of part of the narrative discourse theory by Genette [12, 13]. In common terms, the techniques include the following transformation types: temporal ordering of events, adding supplemental events for story, omitting events, repeating events, and inserting descriptions of characters, objects, or locations. The system selects one or more techniques to generate a discourse on the basis of a generative goal represented as a set of parameters corresponding to the following ten structural features: "supplement," "complexity," "suspense," "length," "hiding," "descriptiveness," "repetition," "diffuseness," "implication," and "temporal independency." Each parameter takes a value of 1 (small), 2 (medium), or 3 (large). Different discourse structures are generated depending on the selected techniques and target parts in the tree structure. As each target part is decided randomly by using several conditions, structures generated by the same set

\footnotetext{
${ }^{*}$ However, it will be possible to synthesize both precise generation of a narrative and diverse narrative generation using INGS in the future. This idea is related to the concept of "fluidity and fixation" in the expanded literary theory [11]. Basically, fluidity and fixation respectively correspond to diverse and precise narrative generation. The concept of fluidity and fixation is one of the basic principles for directing the design and development of the entire INGS. This paper considers the aspect of fluidity or diverse narrative generation.
} 
of parameters are relatively less different from each other.

The input to the system consists of a story, initial generative parameters, total number of discourses generated, and several configurations. Through an iterative generation process, the system outputs a series of discourses. A narrative text from the natural language generator and $\log$ data accompanies each discourse. Figure 1 shows the composition of a series of discourses, where " $\mathrm{D}$ " indicates a discourse. The generation process is summarized as a repetition of the following two phases until the total number of generated discourses reaches the input number: (a) The system iteratively generates discourses based on the same set of parameters. That is, this phase generates a group of relatively similar discourses. The number of discourses in a group is indefinite but loosely controlled, depending on the initial configurations. (b) The system randomly changes the value of a generative parameter and generates a discourse. This phase is called "deviation," wherein a change is made in the discourses generated, which then leads to the generation of a new discourse group in the next phase (a).

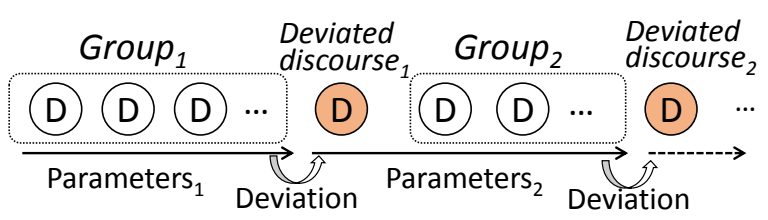

Figure 1: Composition of a sequence of generated discourses

Table 1: Metrics of DFVs [14]

\begin{tabular}{|c|c|}
\hline DFVs & Metrics \\
\hline $\mathrm{DFV}_{1}$ : supplement & $\begin{array}{l}\text { The number of "events" that are positioned outside the story's temporal range and } \\
\text { provide complementary information. }\end{array}$ \\
\hline $\mathrm{DFV}_{2}$ : complexity & $\begin{array}{l}\text { The number of "relations" required for defining temporal order transformation. A } \\
\text { relation nested under one or more relations of temporal ordering is additionally } \\
\text { weighted in proportion to the depth of nesting. }\end{array}$ \\
\hline $\mathrm{DFV}_{3}$ : suspense & $\begin{array}{l}\text { The number of "events" moved from a backward position in the story and not } \\
\text { accounted for before. }\end{array}$ \\
\hline $\mathrm{DFV}_{4}$ : length & The total number of "events" and "descriptions." \\
\hline $\mathrm{DFV}_{5}$ : hiding & $\begin{array}{l}\text { The number of "events" that are contained in the story but not accounted for in the } \\
\text { discourse. }\end{array}$ \\
\hline $\mathrm{DFV}_{6}$ : descriptiveness & The number of "descriptions." \\
\hline $\mathrm{DFV}_{7}$ : repetition & The sum of the numbers of repetition of each "event." \\
\hline $\mathrm{DFV}_{8}$ : diffuseness & $\begin{array}{l}\text { The subtraction of DFV } 5 \text { ("hiding") from DFV } 7 \text { ("repetition"), i.e., "diffuseness" is } \\
\text { increased by "repetition" and decreased by "hiding." }\end{array}$ \\
\hline DFV9: implication & $\begin{array}{l}\text { The number of "relations" required for defining the prospective temporal order } \\
\text { transformation. }\end{array}$ \\
\hline $\begin{array}{l}\mathrm{DFV}_{10} \text { : } \\
\text { temporal independency }\end{array}$ & $\begin{array}{l}\text { The number of "relations" by using the "achronie" technique, which inserts events } \\
\text { that do not have any temporal connection with the story. }\end{array}$ \\
\hline
\end{tabular}

Table 2. Statistics of the whole set of discourses, Group94, and Group 218

\begin{tabular}{l|c|c|c|c|c|c|c|c|c|c}
\hline \multicolumn{10}{c}{ Whole set of discourses } \\
\hline & DFV $_{\mathbf{1}}$ & DFV $_{\mathbf{2}}$ & DFV $_{\mathbf{3}}$ & DFV $_{\mathbf{4}}$ & DFV $_{\mathbf{5}}$ & DFV $_{\mathbf{6}}$ & DFV $_{\mathbf{7}}$ & DFV $_{\mathbf{8}}$ & DFV $_{\mathbf{9}}$ & DFV $_{\mathbf{1 0}}$ \\
\hline min. & 0 & 0 & 0 & 11 & 0 & 0 & 0 & -9 & 0 & 0 \\
\hline max. & 4 & 50 & 8 & 52 & 10 & 8 & 24 & 20 & 6 & 2 \\
\hline avg. & 2.74 & 8.07 & 0.69 & 28.97 & 3.86 & 3.44 & 6.78 & 2.92 & 1.90 & 1.22 \\
\hline \multicolumn{8}{|c|}{ Group94 (discourses 3486-3522) } \\
\hline min. & 2 & 4 & 0 & 17 & 3 & 0 & 3 & -2 & 3 & 0 \\
\hline max. & 2 & 13 & 2 & 27 & 5 & 0 & 9 & 5 & 5 & 0 \\
\hline avg. & 2.00 & 6.61 & 0.25 & 21.56 & 3.89 & 0.00 & 4.83 & 0.94 & 3.89 & 0.00 \\
\hline \multicolumn{8}{|c|}{ Group 218 (discourses 8018-8059) } \\
\hline min. & 4 & 10 & 0 & 35 & 0 & 2 & 7 & 7 & 3 & 2 \\
\hline max. & 4 & 50 & 5 & 52 & 0 & 7 & 18 & 18 & 6 & 2 \\
\hline avg. & 4.00 & 18.10 & 1.71 & 42.02 & 0.00 & 4.76 & 11.10 & 11.10 & 4.22 & 2.00 \\
\hline
\end{tabular}




\section{Visual Interface}

We design the user's action as the following scenario. First, the user explores narratives to find a valuable narrative. This process consists of a repetition of the following two types of actions: selecting a narrative, and overviewing it in a short time. If the selected narrative does not appear valuable, the user will select another narrative. Otherwise, the user will read it closely. In addition, the user can obtain the text data of a narrative for using it to fulfill some objective, e.g., creating an original narrative by editing the narrative text generated by the system. For allowing such a narrative exploration to the user, we employ visualization as the main method for accessing the narratives generated by the system.

\subsection{Basic method of visualization}

The basic concept of visualization is to arrange visual objects, which represent narratives or their groups, into a two-dimensional space. Narratives are hierarchically organized based on the groups and are positioned into a feature-based space. For the purpose of visualization, each narrative is quantified as a set of structural feature values (discourse feature values: DFVs). We defined ten metrics for DFVs corresponding to the ten parameters in the discourse generation system (Table 1) [14]. These metrics are embedded in the generation system, and DFVs are automatically attached to each discourse. After the generation cycle is finished, a preprocessing program statistically analyzes the DFVs in the whole set of generated discourses and each group. In particular, the minimums, maximums, and averages for the whole set and each of the groups are used in the visualization. Table 2 shows the statistics of the whole set and two groups- Group 94 and Group 218 , for example. Because different DFVs have different scales of measure, they are normalized into a common scale running from 0 to 1 .

Visualization consists of the following three layers: the first layer (L1) arranges the visual objects of discourse groups into the space, the second layer (L2) arranges the visual objects of the discourses in a group into the space, and the third layer (L3) expresses a discourse by using a natural language and structural diagram. Each layer is mutually connected with the upper and/or lower layers via the visual objects. L1 and L2 provide a panoramic view of discourses. The user can explore narratives by selecting the visual objects.

In L1 and L2, two of the DFVs are used for arranging the discourses into a two-dimensional space. We indicate the features of the horizontal and vertical axes as $\mathrm{DFV}_{\mathrm{x}}$ and $\mathrm{DFV}_{\mathrm{y}}$, respectively. Choosing different features for these axes results in different arrangements of the discourses. The default features of $\mathrm{DFV}_{\mathrm{x}}$ and $\mathrm{DFV}_{\mathrm{y}}$ are "length" and "complexity," respectively. The user can change $\mathrm{DFV}_{\mathrm{x}}$ and $\mathrm{DFV}_{\mathrm{y}}$ by using the "up"/“down" and "left"/"right" arrow keys.

The following subsections explain the visualization and functions of each layer. The following visualization examples correspond to a series of 10,000 generated discourses consisting of 271 groups. The input story consisted of 16 events in all, summarized as "a warrior named Ivan rescues a princess who was abducted by a snake." This story is made from an output of the story generation program by [15]. Refer to [7, 10] for the full version of the story.

\subsection{L1 window}

L1 arranges all the discourse groups. Figure 2(a) is a screenshot of the L1 window, which con-tains 271 groups. A group is represented by a blue rectangle with the group number. In the space, the left/lower ends and right/upper ends correspond to the minimums and maximums of $\mathrm{DFV}_{\mathrm{x}} / \mathrm{DFV}_{\mathrm{y}}$ in the whole set, respectively. The area of a rectangle represents the ranges of $\mathrm{DFV}_{\mathrm{x}}$ and $\mathrm{DFV}_{\mathrm{y}}$ in the corresponding group. 


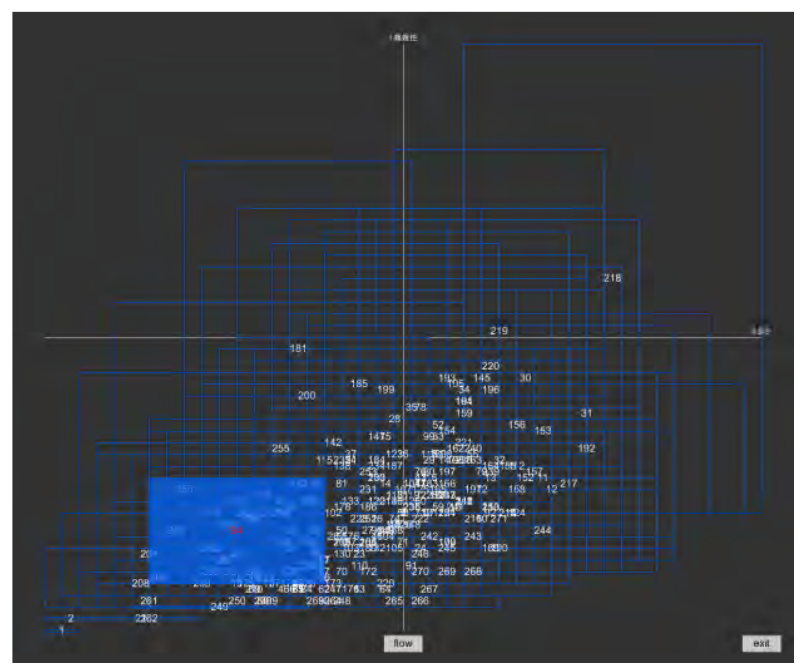

(a) L1 window containing 271 groups

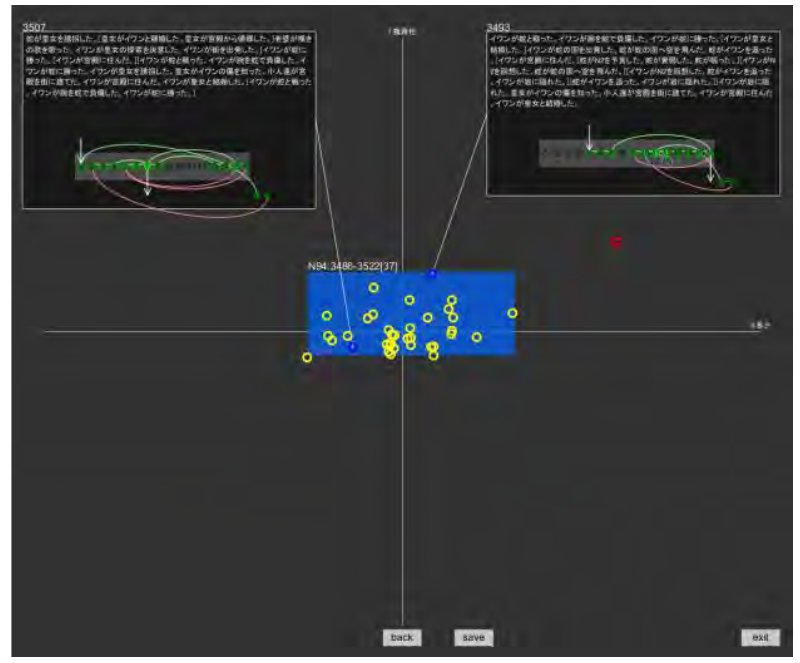

(b) $L 2$ window of Group 94 containing 37 discourses

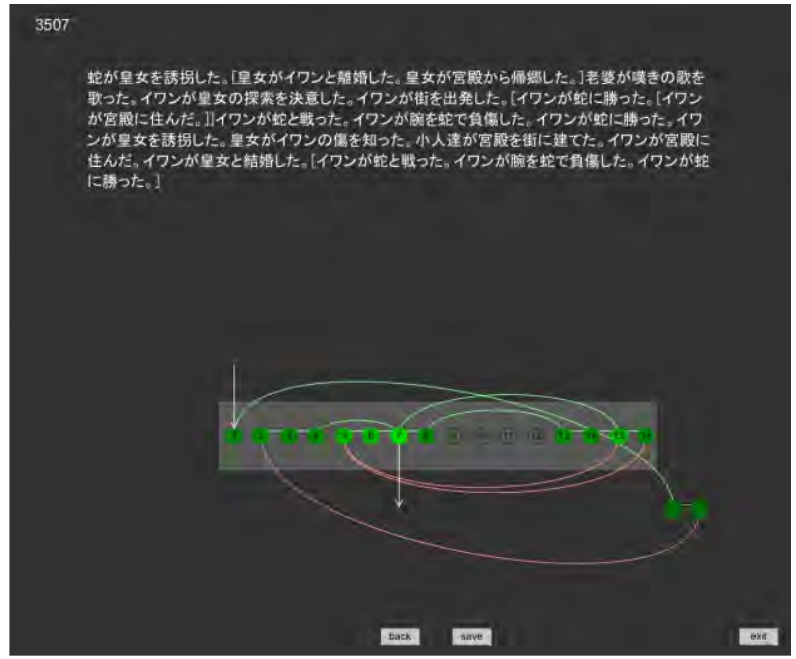

(c) L3 window of

Discourse $_{3507}$

Figure 2: Screenshots of the interface windows

In the $\mathrm{L} 1$ window, the user is allowed to perform the following operations:

- Placing the mouse pointer over a group number to highlight the group object. In Figure 2(a), Group $_{94}$ at the lower left side is highlighted.

- $\quad$ Clicking the left mouse button on a group number to move to the L2 window. 
- Clicking the left mouse button on "flow" to show an animated image of the generated process of the groups.

- Clicking the left mouse button on "exit" to close the interface.

\subsection{L2 window}

L2 arranges the discourses in a selected group. Figure 2(b) shows a screenshot of the L2 window for Group 94 , which contains 37 discourses. A discourse is represented by a small yellow circle. The center of the space-i.e., the intersection of the horizontal and vertical axis lines - corresponds to the average of each $\mathrm{DFV}_{\mathrm{x}}$ and $\mathrm{DFV}_{\mathrm{y}}$. Discourse objects are positioned based on their distance from the center, i.e., the averages. Additionally, the deviated discourse is positioned as a small red circle. This window includes a function to display sub-windows of miniature window L3 for several discourses selected by the user. In Figure 2(b), two sub-windows of Discourse 3493 and Discourse 3507 are shown.

In the L2 window, the user is allowed to perform the following operations:

- Clicking the left mouse button on a discourse object to move to the L3 window.

- Clicking the right mouse button on a discourse object to show a sub-window of the miniature window L3.

- Clicking the left mouse button on "back" to move to the L1 window.

- Clicking the left mouse button on "save" to write the text of the contained discourses to a file.

- Clicking the left mouse button on "exit" to close the interface.

\subsection{L3 window}

L3 expresses a discourse by using Japanese sentences and a diagram of the structure. Figure 2(c) is a screenshot of the L3 window of Discourse ${ }_{3507}$. Figure 3 shows an English translation of the narrative text with commentaries on the discourse structure in angle brackets. The diagram at the lower side represents the discourse structure by using a graph. Each node (numbered circle) corresponds to an event, and the edges show the flow of the narration. Nodes are arranged in the gray box, from left to right, in the order of the story's timeline. Other supplementary events are positioned outside the gray box. The brightness of each node increases in proportion to the recounted number of the event in the discourse, and a dark node represents an event that is not recounted (i.e., it is omitted). Additionally, the edges trace the nodes in the order in which the events are recounted. The edge color indicates the direction; in particular, green and red mean forward and backward, respectively. In addition, the two white arrows indicate the head and tail events in the discourse. The insertion of a description is represented by a cross mark on the midpoint of the edge between the previous and next events.

In the $\mathrm{L} 3$ window, the user is allowed to perform the following operations:

- Clicking the left mouse button on "back" to move to the L2 window.

- Clicking the left mouse button on "save" to write the text to a file.

- Clicking the left mouse button on "exit" to close the interface.

A snake abducted a princess at a garden. [<future $>$ The princess divorced with Ivan. The princess returned home from a palace.] An older woman sang a grief-stricken song at the garden. Ivan resolved to search the princess (at a town). Ivan departed from the town. [<future> Ivan won against the snake at the snake's land. [<future> Ivan lived at a palace.]] Ivan battled with the snake (at the snake's land). Ivan injured his arm by the snake. Ivan won against the snake. Ivan abducted the princess. The princess knew Ivan's wound. Dwarfs built a palace at the town. Ivan lived at the pal-ace. Ivan married with the princess. [<backward> Ivan battled with the snake (at the snake's land). Ivan injured his arm by the snake. Ivan won against the snake.]

Figure 3: An English translation of Discourse 3507 


\section{Discussion}

This section discusses the effect and availability of the above visual interface from several perspectives. First, for showing the actual difference of narratives (i.e., discourse structures) corresponding to the spatial position, we provide another example of L2 visualization for Group 218 with the position in $\mathrm{L} 1$ space (Figure 4). $\mathrm{DFV}_{\mathrm{x}}$ and $\mathrm{DFV}_{\mathrm{y}}$ are the same as in the previous example. The previous example (i.e., Group 94 in Figure 2(b)) is positioned at the lower left side in the L1 space. This indicates that discourses in the group commonly have relatively less "length" and "complexity." The diagrams in the sub-windows help the user to overview several discourses. In the discourses, parts of story events are commonly omitted and several temporal ordering techniques are used. At the same time, there exist differences in the omitted parts and temporal order among the discourses. In contrast, as shown in Figure 4, Group 218 is positioned at the upper right side in the L1 space. The discourses in Group ${ }_{218}$ commonly have long and intricate structures, and all events in the story are recounted. As shown above, the user can grasp macroscopic differences among the groups in the L1 window and microscopic differences in the L2 window.

While designing narrative generation systems, we broadly categorize the users as follows: readers - who appreciate narratives, and authors - who use the system for creating original narratives. When the user explores the narratives as a reader, the visualization helps to lead $\mathrm{him} /$ her to some preferred or required narratives. However, the types of features or information that serves as useful hints to the user require some consideration. The proposed interface uses relatively low-level features for the visualization; high-level features, such as themes, subjects, and styles, may generally be considered when people select narratives. This type of consideration is necessary in designing and upgrading the visualization method.

When the user explores narratives as an author, the system provides materials or ideas for composing original narratives. The discourse generation system can produce many different discourse structures apart from those pertaining to typical commonsensical styles or existing narrative genres, as we quantitatively analyzed in [14]. An advantage of the system's generation ability is its potential to provide unexpected but valuable narratives to the user. The arrangement of narratives on the basis of low-level features will provide useful information for the user. For example, the user will learn a variety of methods to extend a simple story into intricate discourses, as seen in Group 218 (Figure 4) or its neighbor groups.

In modern society, many individuals create and deliver a variety of narratives mainly on the web. Information technology has been aiding the expansion of such individual creative activity
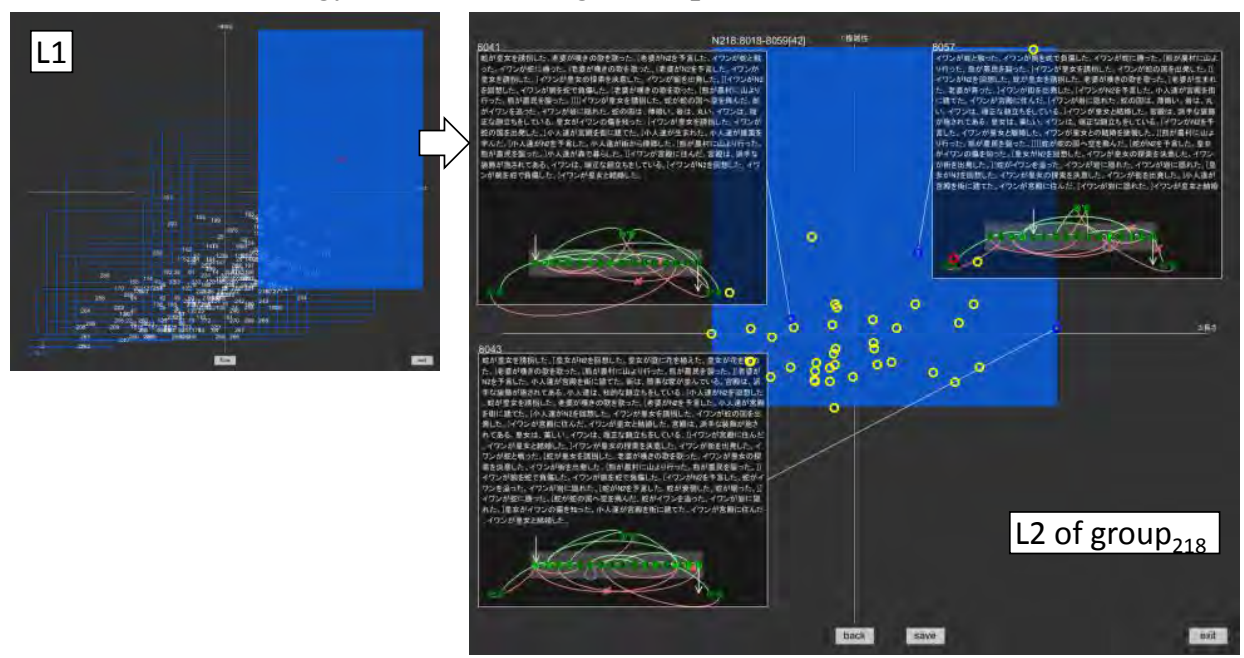

Figure 4: L2 window of Group 218 with the position in L1 space 
in the society. The ultimate goal of our study is to develop a connection between narrative generation systems and humans in a co-creative relationship for unifying them and increasing narrative creation activities in the society. In particular, we are seeking a collective creation model, which behaves as a continual chain reaction of producing narratives, among computers and humans, by using produced narratives. Hori et al. [16] discussed AI technology for supporting continual and chained creative activity among humans in society using computer tools with no automatic creation functions. Although this direction is similar to the above vision, we will position an automated computer system, i.e., a narrative generation system, into the chained creation process as an agent with unique narrative creativity. The potential uses of the system from a human's viewpoint are shown through the following situations: a manga (comic) artist gets primitive materials for his works or an amateur author creates derivative works of computer-generated narratives. The reverse process-i.e., computer generates narratives based on human-created narratives - is also included in our future plans.

The visual interface allows practical experiments or evaluations of the system's availability from this viewpoint. In our first attempt, we conducted an experiment for investigating the effects of computer-generated narratives on human's narrative creation by using the proposed interface. In the experiment, 14 human subjects were divided into two groups: A and B. Each subject in group A used our system and created a variety of original scenarios by obtaining narrative texts on the interface and then editing them to make them unique. Each subject in group B also performed the same creation task, but without using our system. We then analyzed the structural difference between the scenarios created by both these groups. The experimental result is as reported in [8].

\section{Conclusion}

This paper proposed a visual interface for allowing the user to explore and browse various computer-generated narratives. The interface hierarchically visualizes generated narratives into a two-dimensional space based on their structural feature values. The visual arrangement of narratives provides a hint for understanding the similarities and differences among the narratives. With the help of examples of visualized narratives, we have discussed the effect and availability of the visual interface from the perspectives of readers and authors. Despite the fundamental issue of choosing an effective or useful visualization method for the user, the proposed interface allows practical experimentation toward the development of a co-creative relationship among creative computers and people. We have conducted a practical experiment for investigating the effects of computer-generated narratives on humans' narrative creation [8]. In the future, we intend to design a collective narrative generation model as a continual chain reaction between computer narrative generation based on human-created narratives and human narrative creation based on computer-generated narratives.

\section{References}

[1] P. Gervás, "Computational Approaches to Storytelling and Creativity," AI Magazine, vol. 30, no. 3, 2009, pp. 49-62.

[2] M.O. Riedl and V. Bulitko, "Interactive Narrative: An Intelligent Systems Approach," AI Magazine, vol. 34, no. 1, 2013, pp. 67-77.

[3] S. Bringsjord and D.A. Ferrucci, Artificial Intelligence and Literary Creativity: Inside the Mind of BRUTUS, a Storytelling Machine, Lawrence Erlbaum, 1999. 
[4] R. Pérez y Pérez, "The Three Layers Evaluation Model for Computer-Generated Plots," Proc. 5th International Conference on Computational Creativity, 2014.

[5] N. McIntyre and M. Lapata, "Plot Induction and Evolutionary Search for Story Generation," Proc. 48th Annual Meeting of the Association for Computational Linguistics, 2010, pp. 1562-1572.

[6] B. Kartal, J. Koenig, and S.J. Guy, "User-Driven Narrative Variation in Large Story Domains Using Monte Carlo Tree Search," Proc. 13th International Conference on Autonomous Agents and Multiagent Systems, 2014, pp. 69-76.

[7] T. Akimoto and T. Ogata, "A Narratological Approach for Narrative Discourse: Implementation and Evaluation of the System Based on Genette and Jauss," Proc. 34th Annual Conference of the Cognitive Science Society, 2012, pp. 1272-1277.

[8] T. Akimoto and T. Ogata, "An Investigation of Effects of Computer-Generated Narratives on Humans' Narrative Creations," Proc. 32nd Annual Meeting of the Japanese Cognitive Science Society, 2015, pp. 462-471. (in Japanese)

[9] T. Ogata and A. Kanai, Monogatariron no jouhougaku josetsu: Monogatari seisei no sisou to gijutsu wo megutte (An Introduction to Informatics of Narratology: Towards the Thoughts and Technologies of Narrative Generation), Gakubun-sha, 2010. (in Japanese)

[10] T. Akimoto and T. Ogata, "An Information Design of Narratology: The Use of Three Literary Theories in a Narrative Generation System," The International Journal of Visual Design, vol. 7, no. 3, 2014, pp. 31-61.

[11] T. Ogata, "Expanded Literary Theory for Automatic Narrative Generation," Proc. Joint 7th International Conference on Soft Computing and Intelligent Systems and 15th International Symposium on Advanced Intelligent Systems, 2014, pp. 1558-1563.

[12] G. Genette, Narrative Discourse: An Essay in Method, J.E. Lewin (Trans.), Cornell University Press, 1980. (Original work published 1972)

[13] T. Ogata, "An Attempt for Systematization of Narrative Discourse Theory from the Viewpoint of Narrative Generation System," Proc. IPSJ SIG Computers and the Humanities, vol. 99 , no. 85 , 1999, pp. 31-38. (in Japanese)

[14] T. Akimoto and T. Ogata, "Evaluating a Narrative Discourse Generation System Based on Automatic Analyses of a Series of Discourse Structures," Proc. the Workshop on New Directions of Evolutionary Computation and Intelligent Systems, IEEE 2015 International Congress on Evolutionary Computation, 2015, pp. 29-36.

[15] T. Ogata and T. Terano, "Explanation-based Narrative Generation Using Semiotic Theory," Proc. Natural Language Processing Pacific Rim Symposium 91, 1991, pp. 321-328.

[16] K. Hori, M. Akaishi, K. Tanaka, and K. Numa, “AI Technology to Support the Workshops as Fields to Yield Representations," Journal of Japanese Society for Artificial Intelligence, vol. 26, no. 5, 2011, pp. 457-464. (in Japanese) 